\section{Effect of lotrafilcon A silicone hydrogel contact lens on intraocular pressure measurement}

\author{
Carlos García-Resúa, \\ Silvia García-Montero, \\ Hugo Pena-Verdeal, Jorge Gilino, \\ Eva Yebra-Pimentel, Maria J. Giráldez \\ Department of Applied Physics - \\ Optometry Group, University of Santiago \\ de Compostela, Spain
}

\section{Abstract}

The present paper aims to assess the effect of lotrafilcon A contact lenses (CL) in situ on intraocular pressure (IOP) measurement performed with three portable tonometers (ICare, Tonopen and Perkins). This cross-sectional study included thirty young healthy subjects. Intraocular pressure measurements without CL were performed first, followed by IOP measurements with CL for twenty minutes. ICare IOP measurements obtained with lotrafilcon A CL overestimated IOP values without CL by $1 \mathrm{mmHg}$ $(\mathrm{P}<0.001)$. However, both techniques displayed close level of agreement (95\% LoA, -4.17 to +1.63 $\mathrm{mmHg}$ ). Also, differences between both methods tended not to increase $(\mathrm{P}=0.9)$. No significant differences were observed between IOP measurements without and with CL for Perkins (paired- $t$ test, $\mathrm{P}=0.23$ ) and Tonopen XL (paired$t$ test, $\mathrm{P}=0.17$ ). In conclusion, adequate IOP measurements through zero power lotrafilcon A silicone hydrogel CL can be obtained with all three tonometers in healthy eyes, although, in case of ICare, the practitioner must be aware of the $1 \mathrm{mmHg}$ overestimation with CL.

\section{Introduction}

Measurement of intraocular pressure (IOP) is an important part of the ocular examination since high IOP is a significant factor of suffering glaucoma. ${ }^{1}$ Furthermore, assessment of IOP may be necessary in patients wearing soft contact lenses (CL), ${ }^{2,3}$ which must require removal of the $\mathrm{CL}$ and spend some minutes to obtain an accurate measurement. ${ }^{2,3}$

The use of therapeutic CL in clinical practice is widespread. Contact lenses are used primarily in the treatment of corneal disease to relieve pain, protect the cornea from mechanical trauma, to act as a splint to treat lacerations and small perforations, enhance corneal healing and improve corneal hydration. ${ }^{4}$ They may also be used to relieve irregular corneal surfaces, thereby improving visual acuity. ${ }^{2}$ However, therapeutics CL removal to perform IOP measurement is not recommended in many patients, because these lenses must remain in place for extended periods of time, ${ }^{2}$ especially in patients for whom frequent lens insertion and removal may be associated with epithelial trauma, pain, and a potential increase in infection risk. ${ }^{5}$ Portable, handheld tonometers have the advantage of being easily transported from site to site for screening examinations and for those patients for whom the use of a chin rest is difficult. They are especially useful when patients cannot move from their home or during the determination of the daily curve of IOP. ${ }^{6}$

The Perkins applanation tonometer (Medtronic Solan, Jacksonville, FL, USA) is a portable version of Goldman Applanation tonometer (GAT), also requiring topical instillation of fluorescein and anesthesia. Close agreement has previously been found between both tonometers. ${ }^{7}$ For that reason, the Perkins was also considered as the reference tonometer when comparing with portable tonometers, such as the gold standard GAT. Tonopen XL (Medtronic Solan), a handheld tonometer using the same principle as the Mackay-Marg tonometer, ${ }^{8}$ is considered as a good alternative to applanation tonometry, especially for screening purposes. ${ }^{3}$ The ICare (Tiolat Oy, Vantaa, Finland) is a portable tonometer that measures the IOP based on processing the rebound movement of a rod probe, resulting from its interaction with the eye. ${ }^{9}$ Several studies have shown that there is good agreement with respect to $\mathrm{GAT},{ }^{10}$ the NCT Pulsair 3000,1 and also with two portable tonometers such as the Tonopen and Perkins. ${ }^{9}$ Previous studies have investigated the effect of soft CL in IOP measurement using different techniques, such as Goldmann tonometer, $2,3,12,13$ non-contact tonometer, ${ }^{14,15}$ Tonopen $^{3,16-18}$ and ICare, ${ }^{1}$ showing that IOP readings over thin hydrogel and silicone hydrogel CL can be successfully and safely undertaken. The purpose of this study was to evaluate the accuracy of IOP measurements obtained with three portable tonometers through silicone hydrogel CL, commonly used as therapeutic CL due to their high oxygen permeability (Dk). ${ }^{4}$ Lotrafilcon A silicone hydrogel CL were chosen for this study. These CL present the highest modulus of elasticity, ${ }^{19,20} \mathbf{s o}$, the resistance to applanation (derived by its stiffness) should be higher than that of other silicone hydrogel CL with lower modulus of elasticity.

\section{Materials and Methods}

\section{Subjects}

The present is a cross-sectional study that compares IOP measurements obtained by 3
Correspondence: Maria J. Giraldez, Department of Applied Physics - Optometry Group, University of Santiago de Compostela, Edificio Monte da Condesa (Campus Vida), 15782 Santiago de Compostela, Spain.

Tel. +34.881 .813526 - Fax: +34.881 .813534 .

E-mail:mjesus.giraldez@usc.es

Key words: intraocular pressure, silicone hydrogel contact lens, elasticity, portable tonometer, ICare.

Conflict of interests: the authors declare no potential conflict of interests.

Funding: this study was funded in part by the Carlos III Health Institute (of the Spanish Ministry of Health) through grant FIS-PI $10 / 01098$.

Note: all procedures followed the Declaration of Helsinki and the protocol was reviewed and approved by the Ethics Committee of the University of Santiago de Compostela. Written informed consent was obtained from the subjects after careful explanation of the procedures involved.

Received for publication: 29 July 2013

Revision received: 12 November 2013.

Accepted for publication: 24 December 2013.

This work is licensed under a Creative Commons Attribution NonCommercial 3.0 License (CC BYNC 3.0).

(O) Copyright C. García-Resúa et al., 2014

Licensee PAGEPress, Italy

Optometry Reports 2014; 4:1846

doi:10.4081/optometry.2014.1846

tonometers over the same sample study (paired measurements). The inclusion criteria established that all subjects had to be free of ocular disease, had no complaints of excessive lacrimation, were not taking any medications, and had normal general health. ${ }^{21,22}$ As exclusion criteria, subjects with more than 3D of astigmatism were rejected. ${ }^{21}$ With these requirements, a sample study was formed by 30 young adults ( $31 \%$ men and $69 \%$ women) with age ranging from 19 to 32 years (mean age, $20.8 \pm 2.72$ years). After informed consent, measurements of IOP were obtained from the right eye of the entire sample with the three tonometers. All procedures followed the Declaration of Helsinki and the protocol was reviewed and approved by the Ethics Committee of the University of Santiago de Compostela, Spain.

\section{Procedure}

The IOP of only right eyes in each of the volunteers was first measured without the $\mathrm{CL}$; then, IOP was retested 20-30 min following 
insertion of the CL. To reduce inter-observer bias, the same observer carried out all the measurements. At the start of the day each tonometer was calibrated as per the manufacturer's instructions. ICare IOP measurements were performed first followed by Tonopen XL and then Perkins applanation tonometers. ICare was always performed first because its measurement procedure is the only one that does not need anaesthesia. Perkins was always the last because its procedure is the most invasive. To avoid influence of repeated measurements between instruments an interval of 10 min was allowed between each tonometer, which enables to recover from increased aqueous outflow by corneal compression. ${ }^{23}$ After insertion of the CL, 20-30 min were allowed before starting with the measurements for avoiding the effect of managing/removal of CL. ${ }^{16}$ Therefore, the procedure was as follows: ICare without CL - 10 min apart - Tonopen XL without CL - 10 min apart - Perkins without CL - insertion of CL - 2030 min apart - ICare with CL - 10 min apart Tonopen XL with CL - 10 min apart - Perkins with CL. All the measurements were performed between 10:00 and 12:00 $\mathrm{h}$ to minimize the diurnal variation in IOP. ${ }^{6}$

\section{ICare}

The ICare tonometer follows a procedure based on a rebound method. ${ }^{9,24}$ The acquisition process was performed as recommended by the manufacturers. The subject was asked to look straight ahead to a far point while the examiner brought the tonometer near to the subject's eye. Care was taken to ensure that the distance from the tip of the probe to the cornea of the eye was 4 to $8 \mathrm{~mm}$, adjusting the forehead support when necessary. Once the tonometer was correctly adjusted, six repeated IOP readings were acquired by lightly pressing the tonometer button. ${ }^{9}$

\section{Tonopen XL}

Before each measurement without CL, one drop of anaesthetic solution $(1 \mathrm{mg} / \mathrm{mL}$ tetracaine and $4 \mathrm{mg} / \mathrm{mL}$ oxibuprocaine) was instilled on the subject's eye, but no anaesthetic was used with CL in situ. The Tonopen XL probe tip was covered with a new latex tip cover. Four individual measurements were taken by slightly touching the central cornea. The instrument automatically averaged the four readings obtained. In addition to the mean value, the Tonopen XL also displays the variance of the measurements and, according to previous reports, ${ }^{25}$ only those readings within a variance of $5 \%$ were accepted.

\section{Perkins applanation tonometer}

Before acquisition without CL, one drop of fluorescein anaesthetic solution $(2.5 \mathrm{mg} / \mathrm{mL}$ oxibuprocaine and $4 \mathrm{mg} / \mathrm{mL}$ fluorescein) was instilled. Care was taken to obtain an appropri- ate width for the fluorescein rings. Broad fluorescein rings were not used because they could lead to an overestimation of the IOP reading up to $4.6 \mathrm{~mm} \mathrm{Hg.}{ }^{21}$ The use of an additional drop of anaesthetic was also avoided because low fluorescein concentration can underestimate the IOP from 1.5 to $9.0 \mathrm{~mm} \mathrm{Hg}{ }^{21}$ For Perkins with CL procedure neither topical anaesthesia nor fluorescein was used. ${ }^{3}$ Three successive measurements were obtained and then averaged. The bi-prism was disinfected with $3 \%$ hydrogen peroxide and rinsed with saline solution among subjects.

\section{Contact lens}

The silicone hydrogel CL used in this study was lotrafilcon A. The specifications of this CL are as follows: power plano, base curve $8.6 \mathrm{~mm}$, diameter $14.00 \mathrm{~mm}$, centre thickness of 0.08 $\mathrm{mm}$ at -3.00 diopters, $\mathrm{Dk} / \mathrm{t}$ value of 175 Barrer/cm and a modulus of elasticity of 1.74 MPa. ${ }^{19,20}$

\section{Statistical analysis}

Data of this cross-sectional study were analyzed using the statistical package SPSS version 19 for Windows (SPSS Inc., Chicago, IL, USA). A regression analysis was used to evaluate the relationship between IOP measurements with and without CL. To assess the level of agreement, the Bland-Altman method was used. ${ }^{26}$ The bias was assessed statistically as the mean of the differences compared with zero by using paired- $t$ test. The $95 \%$ limits of agreement (LoA) were also calculated. That represents the $95 \%$ of probability to obtain those differences between each pair of techniques, whereas if only the standard deviation
(SD) is used, simply the $68 \%$ of probability of those differences is obtained. Limits of agreement were calculated by multiplying SD by 1.96 factor, and then this was added and subtracted from the mean difference [ $\mathrm{LoA}=$ mean of the difference \pm 1.96 (SD)]. The P value for statistical significance was established at 0.05 .

\section{Results}

Intraocular pressure readings of ICare, Perkins and Tonopen XL tonometers (without and with $\mathrm{CL}$ ) in the right eyes of the 30 subjects were obtained and averaged. Those data are provided as means, SDs and minimum and maximum values (Table 1).

Intraocular pressure values without CL showed positive correlation with the IOP values with CL obtained using ICare $(r=0.75$, $\mathrm{P}<0.001)$, Perkins $(\mathrm{r}=0.51, \mathrm{P}=0.01)$ and Tonopen XL ( $\mathrm{r}=0.242, \mathrm{P}=0.027)$. For illustrative purposes, Figure 1 shows the scatterplot and regression line for these comparisons.

Intraocular pressure measurements without and with CL were compared for each tonometer. No significant differences were observed between IOP measurements made using Perkins $v s$ Perkins_CL (paired- $t$ test, $\mathrm{P}=0.23$ ) and Tonopen XL $v s$ Tonopen XL_CL (paired- $t$ test, $\mathrm{P}=0.17$ ), although a lower and a higher with CL IOP value was obtained with Perkins and Tonopen XL, respectively. On the other hand, there was a statistically significant difference between IOP measurements obtained with ICare $v s$ ICare_CL (paired $t$-test, $\mathrm{P}<0.001$ ), showing an overestimation of the IOP measurements when wearing CL. Those

Table 1. Descriptive statistics of the intraocular pressure obtained by ICare tonometer, Tonopen XL and Perkins without contact lens in situ and with contact lens in 30 right eyes.

\begin{tabular}{lccc} 
Type of tonometer $(\mathrm{mmHg})$ & Mean \pm SD & Minimum & Maximum \\
ICare & $13.06 \pm 2.30$ & 9.00 & 18.00 \\
ICare_CL & $14.33 \pm 2.26$ & 10.00 & 19.00 \\
\hline Tonopen XL & $13.50 \pm 3.6$ & 7.00 & 19.00 \\
Tonopen XL_CL & $14.10 \pm 2.71$ & 10.00 & 19.00 \\
\hline Perkins & $12.65 \pm 2.23$ & 9.00 & 17.00 \\
Perkins_CL & $12.10 \pm 1.68$ & 10.00 & 15.00 \\
\hline
\end{tabular}

SD, standard deviation; _CL, with contact lens.

Table 2. Differences between intraocular pressure measured with and without contac lens with each tonometer.

\begin{tabular}{lcccc} 
& Mean difference & SD & P* & $95 \%$ LoA \\
ICare tonometer & -1.27 & 1.48 & $<0.01$ & -4.17 to 1.63 \\
Tonopen XL & -0.60 & 1.87 & 0.17 & -4.26 to +3.06 \\
\hline Perkins tonometer & 0.55 & 1.98 & 0.23 & -3.33 to +4.43 \\
\hline
\end{tabular}

SD, standard deviation; LoA, limits of agreement. *Paired $t$-test. 
data are displayed in Table 2, where mean difference, level of significance and 95\% LoA for differences between IOP measurements without and with CL are indicated.

The $95 \%$ LoA were $\pm 2.90 \mathrm{mmHg}$ for ICare, $\pm 3.66 \mathrm{mmHg}$ for Tonopen XL and $\pm 3.88 \mathrm{mmHg}$ for Perkins IOP, when comparing without and with CL IOP measurements. A percentage of $93.3,80$ and 85 of the differences between IOP values without and with CL fell within \pm 3.0 $\mathrm{mmHg}$ for ICare, Tonopen XL and Perkins, respectively. Furthermore, linear regression analysis of the difference $v s$ mean did not show a statistical relationship for ICare ( $\mathrm{r}=0.01, \mathrm{P}=0.90)$, and Perkins measurements $(\mathrm{r}=0.32, \mathrm{P}=0.17)$, showing similar differences along the entire range of IOP measurements obtained in this study. On the other hand, trend towards a greater difference as IOP increased was observed for Tonopen XL values $(\mathrm{r}=0.49, \mathrm{P}=0.03)$. Plot of differences against mean, as advocated by Bland and Altmann, are displayed in Figure 2, where the mean of differences and the $95 \%$ LoA obtained with each tonometer are shown.

\section{Discussion}

Silicone hydrogel CL are now being used as therapeutic CL due to their high oxygen permeability. ${ }^{412}$ However, the majority of them present a too high modulus of elasticity that can lead to mechanical and other CL-related complications. ${ }^{19,20}$ For the purpose of the study, ICare, Perkins and Tonopen XL IOP measurements were acquired through silicone hydrogel CL to find out if such measurements are comparable to those acquired without CL on. Higher modulus of elasticity is related with higher stiffness of the CL, so silicone hydrogel CL should offer more resistance to deformation than conventional hydrogel CL. This should be especially important when the ICare tonometer is used to acquire IOP measurements, because this tonometer applies a gently impact of the probe against the cornea. ${ }^{9}$ Indeed, that impact is hardly noticeable by the patient, avoiding the need of topic anaesthetic. ${ }^{9}$ Regarding the refractive power, only plano CL were included in this study. The authors are aware that powerful positive lenses affect the IOP measurements, mainly due to their higher thickness. ${ }^{14}$ However, it was reported that there is a wide range of positive lens power (less than +3.00 diopters) and central thickness (less than $0.3 \mathrm{~mm}$ ) that does not affect IOP measurements. ${ }^{14}$ Further studies are needed to analyze this effect.

Silicone hydrogel CL used in this study was lotrafilcon A, because this CL presents the highest modulus of elasticity. ${ }^{19,20}$ So, the effect of this CL on IOP measurements derived by its stiffness should be higher than the effect derived by other silicone hydrogel CL with lower modulus of elasticity. This could be especially relevant when using ICare, because of the gently contact of the probe during the acquisition process. In this sense, Zeri et al. ${ }^{1}$ studied ICare IOP measurements with senofilcon A CL on. In contrast with the present study, they did not observed effect of senofilcon A CL when measuring IOP by rebound tonometry. That may be due to the fact that senofilcon A CL present a much lower modulus of elasticity than lotrafilcon A (senofilcon A: $0.50 \pm 0.04$
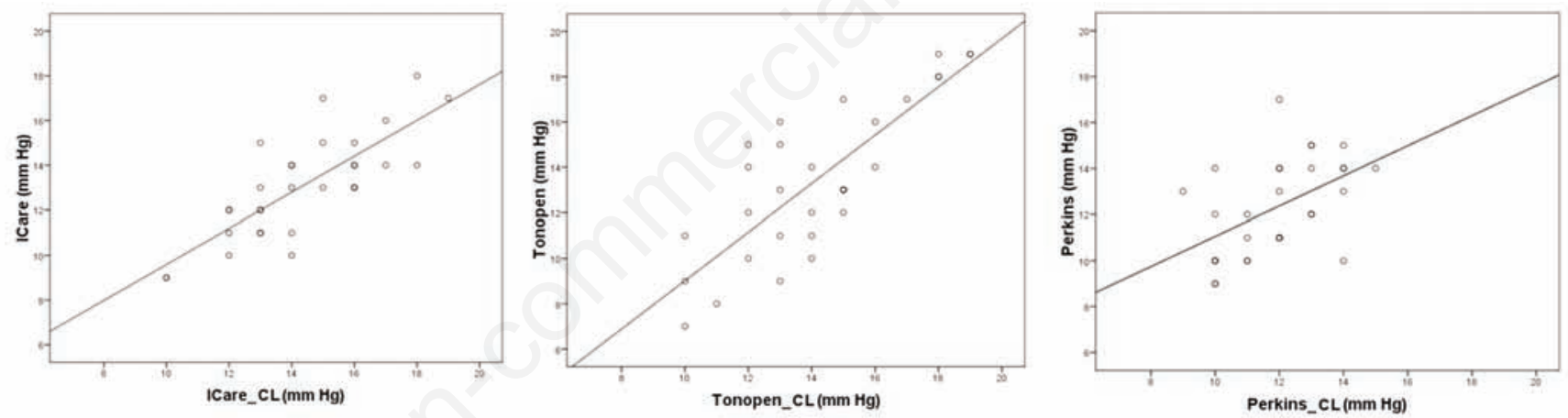

Figure 1. Regression line and scatterplot showing the relationship between ICare, Tonopen and Perkins intraocular pressure measurements with (_CL) and without contac lenses.
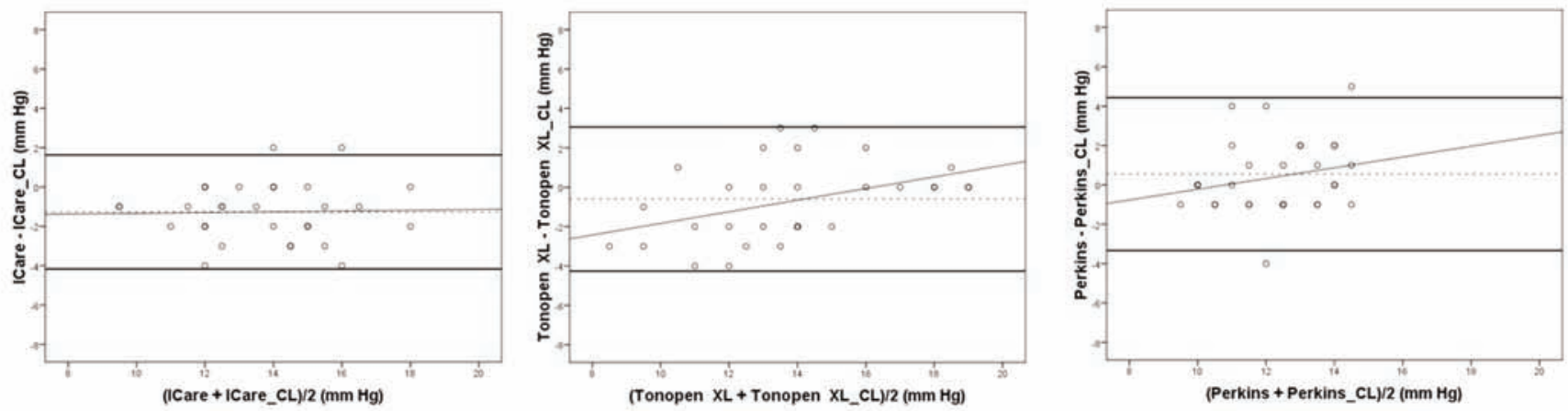

Figure 2. Plots of difference $v s$ mean of intraocular pressure values for ICare, Tonopen and Perkins intraocular pressure measurements with (_CL) and without contac lenses. The mean of the difference, 95\% limits of agreement (solid lines), and line of equality between techniques (dashed line, ICare $\mathbf{r}^{2}<0.001$; Tonopen $\mathbf{r}^{2}=0.10$; Perkins=0.24) are shown. 
MPa $v$ lotrafilcon A: $1.74 \pm 0.15 \mathrm{MPa}) .{ }^{19,20}$ Zeri et al. ${ }^{1}$ used $+2.00 \mathrm{D},-2.00 \mathrm{D}$ and $-6.00 \mathrm{D}$ power CL that should affect IOP measurements more than plano CL employed in our study (specially +2.00D). So, the $1 \mathrm{mmHg}$ mean overestimation for ICare IOP measurements with CL found here is derived by both the highest elastic modulus of lotrafilcon A and the smaller interaction with the cornea offered by ICare tonometer. ${ }^{22,27}$

From a clinical point of view, although ICare showed statistical differences with CL on, those differences were within the clinically acceptable error of $\pm 3.0 \mathrm{mmHg} .{ }^{22,27}$ In a greater extent, the 95\% LoA showed a coefficient of accuracy of $\pm 2.9 \mathrm{mmHg}$, and the $93 \%$ of the differences fell within this clinically accepted range. Although Perkins IOP values did not show differences through lotrafilcon A silicone hydrogel CL, a mean tendency of $0.55 \mathrm{mmHg}$ underestimation was found, which is similar to that previously referred for Goldmann tonometry with Balafilon A CL. ${ }^{12}$ Studies have shown that the use of Goldman applanation tonometry without fluorescein underestimates the intraocular pressure by $2 \mathrm{mmHg}$ in subjects both with and without CL in situ. ${ }^{3}$ Regarding Tonopen, the effect of soft CL on IOP measurements was previously studied, ${ }^{3,16-}$ ${ }^{18}$ although non-silicone hydrogel was evaluated. In this study the effect of silicone hydrogel CL on Tonopen IOP measurements was evaluated and no statistical significant differences were found with CL in situ. Taking into account that $95 \%$ of differences between IOP measurements with and without CL were within $\pm 3.0 \mathrm{mmHg}$ clinical margin, ${ }^{22,27}$ wen can derive that Tonopen offers similar results when wearing other silicone hydrogel CL.

Another important point was to analyze the differences over the entire range of the IOP studied, so a plot of mean and differences between both methods, as advocated by Bland and Altmann, ${ }^{26}$ were done (Figure 2). IOP differences when using CL were uniform thorough the IOP range for ICare and Perkins tonometers, and that was confirmed by a regression analysis between the mean and the difference. So, in the range of the IOP measured here, we can predict that IOP ICare measurements with silicone hydrogel CL in situ show a mean overestimation of $1 \mathrm{mmHg}$, with a probability of $95 \%$ that this overestimation is less than $4.0 \mathrm{mmHg}$, whereas for Perkins measurements this maximum difference could be up to $4.4 \mathrm{mmHg}$. On the other hand, Tonopen showed a significant tendency between mean and differences $(r=0.49$, $\mathrm{P}=0.03$ ) and, as can be seen in Figure 2, for lower IOP values, Tonopen with CL in situ overestimated IOP measurements without CL, whereas for higher IOP values this trend was the opposite. This makes it difficult to predict the IOP value when Tonopen measurements are done with CL on.

Hence, accurate IOP measurements through silicone hydrogel CL can be obtained with the rebound ICare tonometer in healthy eyes. This means that it is not necessary to remove the lotrafilcon A CL during the IOP reading. The practitioner must be aware of the $1 \mathrm{mmHg}$ overestimation when using ICare, although in primary care this is not so critical (because it is focused in screening purposes). Nonetheless, care should be taken when anomalous IOP values are obtained.

\section{Conclusions}

Future studies including eyes with corneal pathologies, glaucoma and/or CL of high plus power are needed to analyze the value of IOP measured with CL on.

\section{References}

1. Zeri F, Calcatelli P, Donini B, et al. The effect of hydrogel and silicone hydrogel contact lenses on the measurement of intraocular pressure with rebound tonometry. Cont Lens Anterior Eye 2011;34:260-5.

2. Scibilia GD, Ehlers WH, Donshik PC. The effects of therapeutic contact lenses on intraocular pressure measurement. CLAO J 1996;22:262-5.

3. Lim L, Ng TP, Tan DT. Accurate intraocular pressure measurement in contact lens wearers with normal pressures. CLAO J 1997;23:130-3.

4. Lim L, Tan DT, Chan WK. Therapeutic use of Bausch \& Lomb PureVision contact lenses. CLAO J 2001;27:179-85.

5. Kanpolat A, Ucakhan 00. Therapeutic use of Focus Night \& Day contact lenses. Cornea 2003;22:726-34.

6. Wilensky JT, Gieser DK, Dietsche ML, et al. Individual variability in the diurnal intraocular pressure curve. Ophthalmology 1993;100:940-4.

7. Wingert TA, Bassi CJ, McAlister WH, et al. Clinical evaluation of five portable tonometers. J Am Optom Assoc 1995;66:670-4.

8. Moses RA, Marg E, Oechsli R. Evaluation of the basic validity and clinical usefulness of the Mackay-Marg tonometer. Invest Ophth Visual 1962;1:78-85.

9. Garcia-Resua C, Gonzalez-Meijome JM, Gilino J, et al. Accuracy of the new ICare rebound tonometer vs. other portable tonometers in healthy eyes. Optometry Vision Sci 2006;83:102-7.

10. Poostchi A, Mitchell R, Nicholas S, et al. The iCare rebound tonometer: comparisons with Goldmann tonometry, and influence of cen- tral corneal thickness. Clin Exp Ophthalmol 2009;37:687-91.

11. Kontiola A, Puska P. Measuring intraocular pressure with the Pulsair 3000 and Rebound tonometers in elderly patients without an anesthetic. Graef Arch Clin Exp 2004;242:3-7.

12. Allen RJ, Dev Borman A, Saleh GM. Applanation tonometry in silicone hydrogel contact lens wearers. Cont Lens Anterior Eye 2007;30:267-9.

13. Zeri F, Lupelli L, Formichella P, et al. Goldmann applanation tonometry over daily disposable contact lens: accuracy and safety of procedure. Cont Lens Anterior Eye 2007;30:233-8.

14. Patel S, Illahi W. Non-contact tonometry over soft contact lenses: effect of contact lens power on the measurement of intra-ocular pressure. Cont Lens Anterior Eye 2004;27:337.

15. Patel S, Stevenson G. Influence of lens material and intra-ocular pressure on the outcome of non-contact tonometry over soft contact lenses. Cont Lens Anterior Eye 2009;32:68-72.

16. Khan JA, LaGreca BA. Tono-Pen estimation of intraocular pressure through bandage contact lenses. Am J Ophthalmol 1989;108:422-5.

17. Mark LK, Asbell PA, Torres MA, et al. Accuracy of intraocular pressure measurements with two different tonometers through bandage contact lenses. Cornea 1992;11:277-81.

18. Panek WC, Boothe WA, Lee DA, et al. Intraocular pressure measurement with the Tono-Pen through soft contact lenses. Am J Ophthalmol 1990;109:62-5.

19. Young G, Garofalo R, Harmer 0, et al. The effect of soft contact lens care products on lens modulus. Cont Lens Anterior Eye 2010;33:210-4.

20. Young G, Garofalo R, Peters S, et al. The effect of temperature on soft contact lens modulus and diameter. Eye Contact Lens 2011;37:337-41.

21. Whitacre MM, Stein R. Sources of error with use of Goldmann-type tonometers. Surv Ophthalmol 1993;38:1-30.

22. Holladay JT, Allison ME, Prager TC. Goldmann applanation tonometry in patients with regular corneal astigmatism. Am J Ophthalmol 1983;96:90-3.

23. Krakau CE, Wilke K. On repeated tonometry. Acta Ophthalmol Scan 1971;49:611-4.

24. Kontiola AI. A new induction-based impact method for measuring intraocular pressure. Acta Ophthalmol Scan 2000;78:142-5.

25. Hessemer V, Rossler R, Jacobi KW. Tono-Pen, a new tonometer. Int Ophthalmol 1989;13:516.

26. Bland JM, Altman DG. Statistical methods for assessing agreement between two methods of clinical measurement. Lancet 1986;1:30710.

27. Moses RA, Arnzen RJ. Instantaneous tonometry. Arch Ophthalmol-Chic 1983;101:249-52. 